\title{
MTA1 Overexpression Induces Cisplatin Resistance Innasopharyngeal Carcinoma by Promoting Cancer Stem Cells Properties
}

\author{
Xiaohua Feng ${ }^{2,8}$, Qianbing Zhang ${ }^{3,8}$, Songxin $\mathrm{Xia}^{4,8}$, Bing Xia ${ }^{7}$, Yue Zhang ${ }^{5,7}$, Xubin Deng ${ }^{5}$, Wenmei Su ${ }^{6, *}$, \\ and Jianqing Huang ${ }^{1, *}$
}

\begin{abstract}
Themetastasis-associated gene 1 (MTA1) oncogene hasbeen suggested to be involved in the regulation of cancer progression. However, there is still no direct evidence that MTA1 regulates cisplatin (CDDP) resistance, as well as cancer stem cell properties. In this study, we found that MTA1 was enriched in CNE1/CDDP cells. Knock down of MTA1 in CNE1/CDDP cells reversed CSCs properties and CDDP resistance. However, ectopic expression of MTA1 in CNE1 cells induced CSCs phenotypes and CDDP insensitivity. Interestingly, ectopic overexpression of MTA1-induced CSCs properties and CDDP resistance were reversed in CNE1 cells after inhibition of PI3KIAkt by LY294002. In addition, MTA1 expression and Akt activity in CNE1/CDDP cells was much higher than that in CNE1 cells. These results suggested that MTA1 may play a critical role in promoting CDDP resistance in NPC cells by regulatingcancer stem cell properties via thePI3KIAkt signaling pathway. Our findings suggested that MTA1 may be a potential target for overcoming CDDP resistance in NPC therapy.
\end{abstract}

\section{INTRODUCTION}

Nasopharyngeal carcinoma (NPC) is one of the most prevalent

${ }^{1}$ Department of Medical Oncology, Affliated Cancer Hospital of Guangzhou Medical University; Cancer Center of Guangzhou Medical University (CCGMU), Guangzhou, China, ${ }^{2}$ Department of Otolaryngology, General Hospital of Guangzhou Command, Guangzhou, China, ${ }^{3}$ Cancer Institute of Southern Medical University, Guangzhou, China, ${ }^{4}$ Department of stomatology, Guangdong Provincial Hospital of Traditional Chinese Medicine, Guangzhou, China, ${ }^{5}$ Department of Radiotherapy, Nanfang Hospital of Southem Medical University, Guangzhou, China, ${ }^{6}$ Cancer Center of Affiliated Hospital of Guangdong Medical College, Zhanjiang, China, ${ }^{7}$ Department of Cardiology, $458^{\text {th }}$ Hospital of People's Liberation Army, Guangzhou, China, ${ }^{8}$ These authors contributed equally to this work. *Correspondence: JianqingHuang_01@126.com (JH);

WenmeiSu@126.com (WS)

Received 10 February, 2014; revised 3 August, 2014; accepted 5 August, 2014; published online 22 September, 2014

Keywords: cancer stem cell, cisplatin-resistance, MTA1, nasopharyngeal carcinoma forms of cancer in Southern Asia, though is rare in other part of the world (Spano et al., 2003). Cisplatin (CDDP) is one of the most effective and widely used anticancer drugsagainst various human cancers including NPC (Serin et al., 1999). However, CDDP resistance usuallydevelops during NPC therapy and becomes a major obstacle for the effective treatment of NPC (Ma et al., 2010).

The cancer stem cell theory has been well accepted to explain the tumor heterogeneity and thecarcinogenesis process. The cancer stem cells (CSCs) are cellswithin a tumor that possess the capacity to self-renew and differentiate into the heterogeneous lineages of cancer cells that comprisethe whole tumor(Singh et al., 2003). Increasing studiesshowed thatdrug-resistant cancercells display a stem-like signature(Donnenberg and Donnenberg, 2005; Vinogradov and Wei, 2012).

Increasing evidence has indicated that the humanmetastasisassociated gene 1 (MTA1) is a key factor in tumormetastasis(Salot and Gude, 2013). The MTA1protein has been found in the nucleosome remodelinghistone deacetylase (NuRD) complex, which has been shown tomodify or remodel chromosomes(Zhang et al., 1999). As an important regulator of cell fate with a role in theoncogenesis and progression of many malignant tumors, MTA1has attracted widespread attention(Kumar et al., 2003; Li et al., 2012a). Despite the intensive study on MTA1, the role of MTA1 in CDDP resistance has not been investigated.

\section{MATERIALS AND METHODS}

\section{Cell lines andreagents}

CNE1 cell line was maintained in RPMI-1640 medium containing $10 \%$ FBS, $2 \mathrm{mM}$ glutamine and $10 \mathrm{mM}$ HEPES at $37^{\circ} \mathrm{C}$ and $5 \%$ $\mathrm{CO}_{2}$. CNE1/CDDP cell line was developed by continuous exposure toCDDP starting at $0.1 \mu \mathrm{mol}$ and increasing in a stepwise manner to $10 \mu \mathrm{mol}$. CDDP, LY294002 (PI3K inhibitor) and Hoechst 33342 were purchased from Sigma (USA). The MTA1, p-Akt, AKT primary antibodies and horseradish peroxidase (HRP)-labeledanti-rabbit secondary antibody were purchased from Cell Signaling Technology (USA).

\section{MTT assay}

The MTT assay was carried out according to previous study ( $\mathrm{Li}$ et al., 2012b). 
A

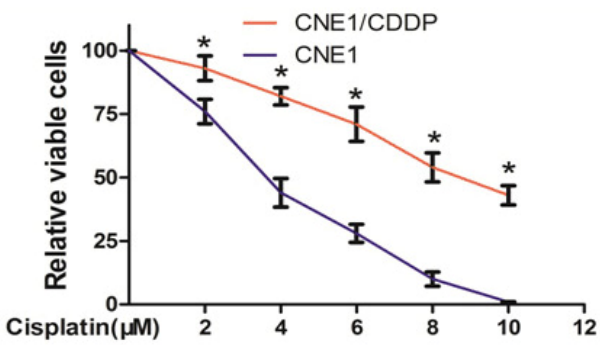

B

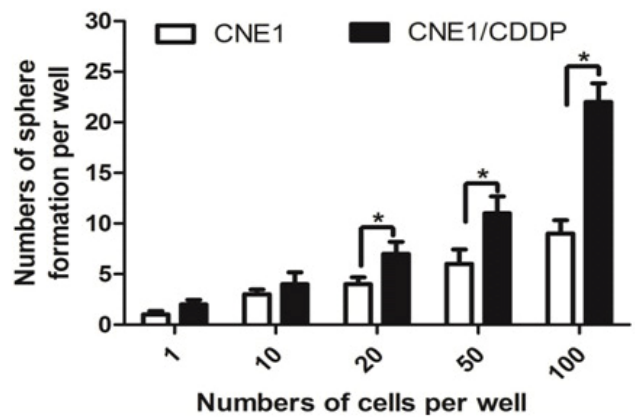

C
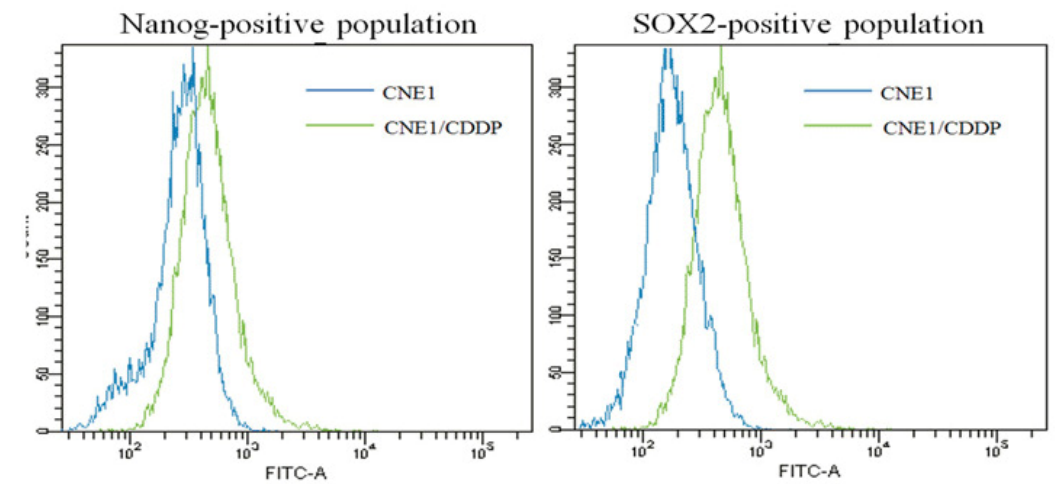

Fig. 1. (A) Compared with the parental CNE1 cells, CNE1/CDDP cells were resistant to CDDP. (B) The diluted sphere forming assay showed that the self-renewal ability was significantly higher in CNE1/CDDP cells than that in their parental CNE1 cells. (C) The FACS analysis showed that SOX2- and Nanog-positive cell populations were higher in CNE1/CDDP cells than that in their parental CNE1 cells.

Tumorsphere culture and serial passageassay

Tumor sphere culture was performed as described previously(Singh et al., 2003). Single cells were plated in Ultra Low Attachment plates (Corning) in serum-free DMEM-F12 supplemented with $10 \mathrm{ng} / \mathrm{ml} \mathrm{bFGF}, 10 \mathrm{ng} / \mathrm{ml}$ EGF, and B27 (all from Invitrogen). In these conditions cells grew as suspension spherical clusters. The serial diluted sphere formingassay was performed as previously described (Liu et al., 2014).

\section{Flow cytometry analysis}

The following antibodies were used for flow cytometry analysis:SOX2-PE (R\&D Systems) and Nanog-PE (R\&D Systems). The analysis for SOX2 and Nanog expression in NPC CSCs was carried out as previouslydescribedwith the addition of SOX2-PE or Nanog-PE (Venkatesha et al., 2012).

Lentivirus production and transduction

shRNA lentiviral particles (sh-MTA1, sh-Ctrl) were from GeneChem (China). MTA1 overexpression lentiviral particles were purchased from purchased from GeneCopoeia, Inc. Cellswere incubated with the lentiviral particlesin presence of hexadimethrine bromide $(8 \mu \mathrm{g} / \mathrm{ml})$. Transduced cells were selected in puromycincontaining media.

\section{Western blotting}

Total cell lysates were separatedby SDS/PAGEand transferred to PVDF membrane.The blotted membrane was then blocked with 3\% BSA for $1 \mathrm{~h}$ at roomtemperature and incubated overnightat $4^{\circ} \mathrm{C}$ with primary antibodies as follows: MTA1, p-Akt and AKT. Membranes were probed withhorseradish peroxidase (HRP)-labeled anti-rabbit secondary antibody. The bolted membrane was detected by enhancedchemiluminescence detection kit (ECL) (Amersham, UK).
Real-time reverse transcription-polymerase chainreaction (qPCR) analysis

Total RNA was extracted from the cellsusing Trizol plus kit (TaKaRa, Japan). The isolated RNA was used for cDNA synthesis using reverse transcription reaction with Superscript II RNaseH Reverse Transcriptase (Invitrogen) and random hexamers (Promega). Real-time PCR was performed using aStratagene Mx3000 Real-Time PCR System and Brilliant SYBR Green QPCR Master Mix (Stratagene). The primers were following: ABCG2,5'-agctccgatggattgccag-3'(forward) and 5'-gagggttcccga gcaagttt-3'(reverse); SOX2, 5'-atggagaaaacccggtacgc-3'(forward) and5'-tttgcgtgagtgtggatgg-3' (reverse);Nanog,5'-ggacac tggctgaatccttcc-3'(forward) and 5'-tcccatgcattcaaactgagg-3' (reverse).

\section{Xenograft studies}

A total of $2 \times 10^{3}$ CNE1/CDDP-sh-Ctrl or CNE1/CDDP-sh-MTA1 cells were subcutaneously inoculated into the back of nude mice. When established tumours of approximately 10 mmin diameter were detected,mice wererandomised into four groups. The mice were treated with CDDP (5 mg/kg, two times/week, i.p.) or DMSO. Tumor volumes were measured every week and calculated with the formula: $\left(\mathrm{mm}^{3}\right)=\left(\mathrm{L} \times \mathrm{W}^{2}\right) \times 0.5$. The tumor growthinhibitory effects were examined for about 4 weeks.

\section{Statistical analysis}

The Student's $t$-test was used to evaluate the statistical significance of the results. All $P$ values represent two-sided tests of statistical significance. All analyses wereperformed with the SPSS 13.0 software package. $\mathrm{P}<0.05$ was considered statistically significant. 
A

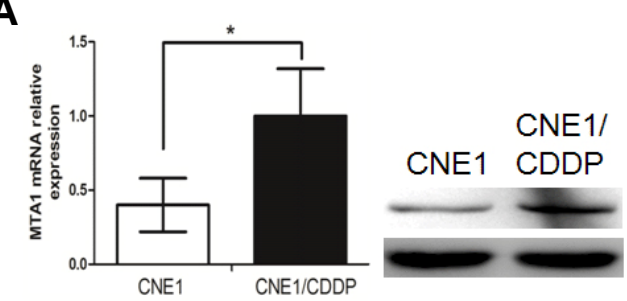

C

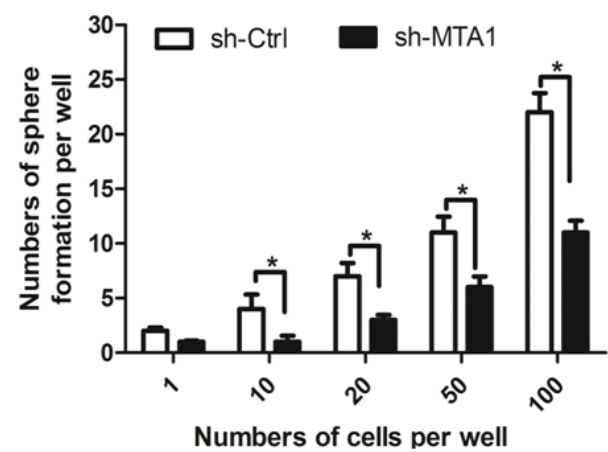

B

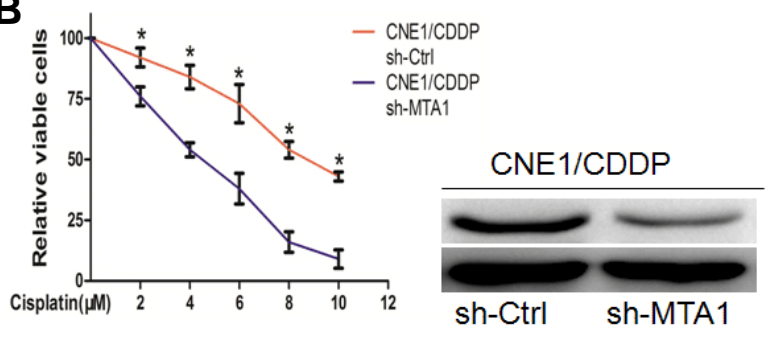

D

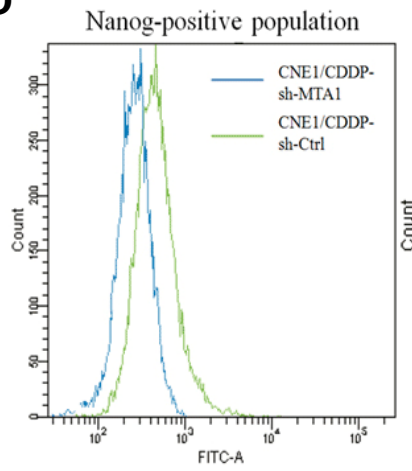

SOX2-positive population

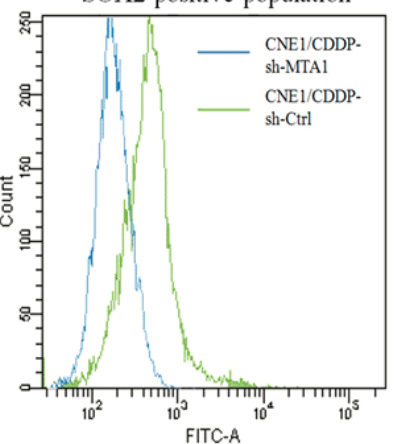

Fig. 2. (A) Both the mRNA and protein levels of MTA1 in CNE1/CDDP cells were significantly higher than those in their parental cells. (B) Knockdown of MTA1 in CNE1/CDDP cells overcome CDDP resistance. (C) Knockdown of MTA1 expression reduced the self-renewal ability of CNE1/CDDP cells. (D) Knockdown of MTA1 reduced the SOX2- and Nanog-positive cell populations in CNE1/CDDP cells.

\section{RESULTS}

\section{CDDP resistance in CNE1/CDDP cells correlates with CSCs} property

We first established a CDDP-resistant NPC cell lineCNE1/CDDP by continuous exposure to CDDP startingat 0.1 $\mu \mathrm{mol}$ and increasing in a stepwise manner to $10 \mu \mathrm{mol}$. We found that established CNE1/CDDP cells were resistant to CDDP, when compared with the parental CNE1 cells (Fig. 1A). The serial diluted sphere formingassay showed that self-renewal ability of CNE1/CDDP cells was much stronger than that of the CNE1 cells (Fig. 1B). The SOX2- and Nanog-positive cellpopulationswere also substantially elevated in CNE1/CDDP cells when compared with those in CNE1 cells, as determined by the flow cytometry analysis (Fig. $1 \mathrm{C}$ ). These results indicate that CDDP resistance development correlates CSCs phenotype in NPC cells.

MTA1 promotes CSCs phenotype and CDDP insensitivity in NPC cells

To explore the effect of MTA1 on CDDP resistance in NPC cells, we first compared the expression levels of MTA1 in CNE1 cells with those in CNE1/CDDP cells at both MRNA and protein levels. We found that both the mRNA and protein levels of MTA1 in CNE1/CDDP cells were significantly higherthan those in their parental cells (Fig. 2A). These results suggest a potential role of MTA1 in CDDP resistance development.

To confirm the role of MTA1 in CDDP resistance in NPC cells, we used the specific stable MTA1 shRNA to inhibit MTA1 expression in $\mathrm{CNE} 1 / \mathrm{CDDP}$ cells. Figure $2 \mathrm{~B}$ showed that knockdown of MTA1 in CNE1/CDDP cellsovercome CDDP resistance. Meanwhile, knockdown of MTA1 expression reduced the selfrenewal ability andSOX2- and Nanog-positive cellpopulations in
CNE1/CDDP cells, respectively (Figs. 2C and 2D). Collectively, these data indicate that MTA1 overexpression induces CDDP resistance in NPC cells probably through promotion of cancer stem-like phenotype.

PI3KIAkt signaling is required for the cancer stem cellpromoting effect of MTA1 overexpression

To explore the mechanism underlying MTA1 overexpressioninduced cancer stem-like phenotype and CDDP resistance in CNE1 cells, the PI3K/Akt pathway inhibitor LY294002 was used. We found that MTA1 overexpression decreased CDDP efficacy, accompanied byincreased self-renewal ability and increased SOX2- and Nanog-positive cell populationsin CNE1 cells. However, these effects were reversed or blocked by additional LY294002 treatment after 48-hincubation (Figs. 3A-3C, respectively).Furthermore, Fig. 3D showed that MTA1 overexpression could significantly stimulate PI3K/Akt signaling as expressed by enhanced levels of p-Akt in CNE1 cells. Combined with that Akt activity in CNE1/CDDP cells was much higher than that in CNE1 cells (Fig. 3E), the above data indicate that MTA1 overexpression induces CSCs phenotype and CDDP resistance via $\mathrm{PI}$ KK/Akt-dependent pathway in CNE1 cells.

MTA1 knockdown inhibites NPC tumorigenesis and increases CDDP sensitivity in CNE1/CDDP xenografttumorharbored nude mice

To determine the effect of MTA1 expression on CDDP sensitivity in vivo, CNE1/CDDP sh-MTA1 and CNE1/CDDP cells were subcutaneously inoculated into the nude mice. After the xenografts in each groupreached the same size (around $10 \mathrm{~mm}$ in diameter), the mice were treated with CDDP ( $5 \mathrm{mg} / \mathrm{kg}$, two times/week, i.p.) or DMSO. We found that both the growth rate and size of the xenografts derived from CNE1/CDDP sh-MTA1 
A

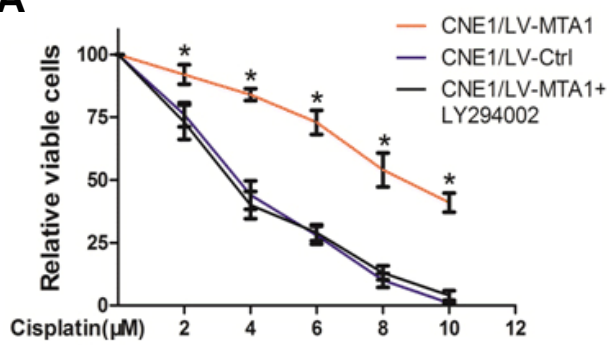

C
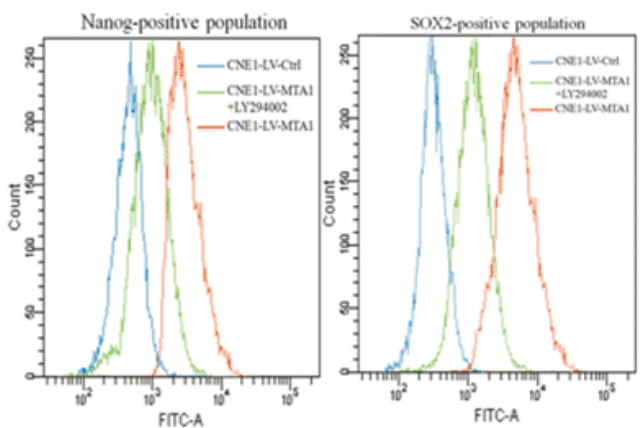

E

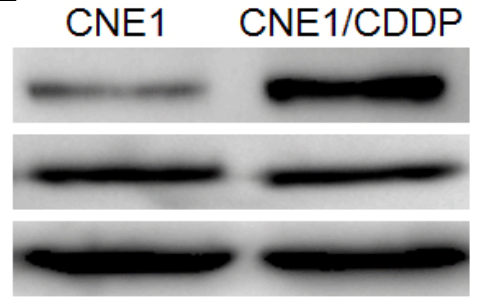

p-AKT

AKT

GAPDH
B

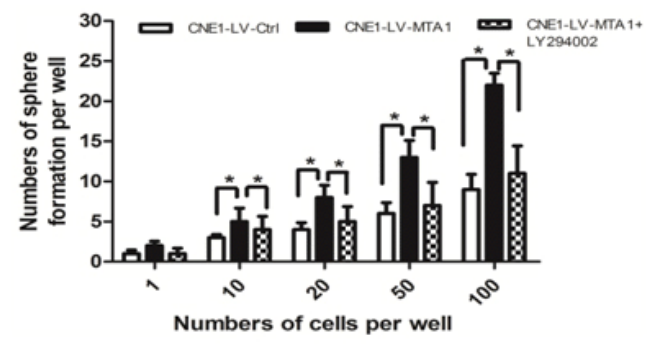

D

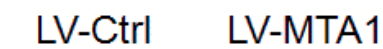

Fig. 3. (A-C) MTA1 overexpression decreased CDDP efficacy, accompanied by increased self-renewal ability and increased SOX2- and Nanogpositive cell populations CNE1 cells. However, these effects were reversed or blocked by additional LY294002 treatment after 48-h incubation. (D) MTA1 overexpression could significantly stimulate PI3K/Akt signaling. (E) Akt activity in CNE1/CDDP cells was much higher than that in CNE1 cells.

cells were much smaller than that from sh-Ctrl treated cells after receiving the same doses of CDDP or CNE1/CDDP sh-MTA1 cells treated with DMSO (Figs. 4A and 4B). These results indicated that knockdown of MTA1 could sensitize tumors to CDDP.

\section{DISCUSSION}

CSCs have been shown to play an important role in the development of chemoresistance in cancer therapy.Resistance of cancer cells to CDDP was shown to be related to cancer stem cell properties (Barr et al., 2013; Shafee et al., 2008). Consistent with the above findings, the present study found that in the established CNE1/CDDP cell line, CDDP resistance correlates with changes in cellular phenotypes and moleclular markers consistent with CSCs.

Growing evidences have shown the close correlation of MTA1 and cancer malignant progression(Nagaraj et al., 2013; Song et al., 2013). Nevertheless, the role of MTA1 in CDDP resistant and CSCs has not been fully explored before. In the present study, we found that CNE1/CDDP cells expressed higher level of MTA1 than CNE1 cells. Knockdown of MTA1 in CNE1/CDDP cells overcome CDDP resistance, consistent with reduced CSCs properties. It was reported that selective down-regulation of
MTA1 in breast cancer cells increased PTEN expression and inhibited stimulation of the PI3K/AKT signaling (Reddy et al., 2012). In addition, MTA1 may lead to increased stabilization of $\beta$ catenin and stimulation of Wnt1 target genes in the murine mammary epithelial and humanbreast cancer cells (Kumar et al., 2010). It is well known that the activated AKT signaling pathway play significant role in CDDP resistance (Wang et al., 2014). We recently found that $A K T / \beta$-catenin signaling played significant role in regulating CSCs in NPC (Ma et al., 2013). We then asked whether MTA1 regulated CDDP resistance through modulating the AKT activity. In our study, after inhibition of PI3K/Akt by LY294002, ectopic overexpression of MTA1-induced CSCs properties and CDDP resistance were reversed in CNE1 cells. In addition, MTA1 expression and Akt activity in CNE1/CDDP cells was much higher than that in CNE1 cells. These results indicate that MTA1 induce CSCs and CDDP resistance dependent on PI3K/Akt pathway. The possible upstream signaling pathways that upregulates MTA1 expression in CNE1/CDDP cells may be complicated. Recently, the Nuclear Factor-kappa B (NF-kB) signaling pathway has been identified as a key player in CDDP- resistance mechanisms (Morais et al., 2010).The CDDP-resistant cells show increased expression of NF- $\mathrm{KB}$, compared to their matched cisplatin sensitive parent, implicating NF- $\mathrm{KB}$ as a potential mediator of 
A

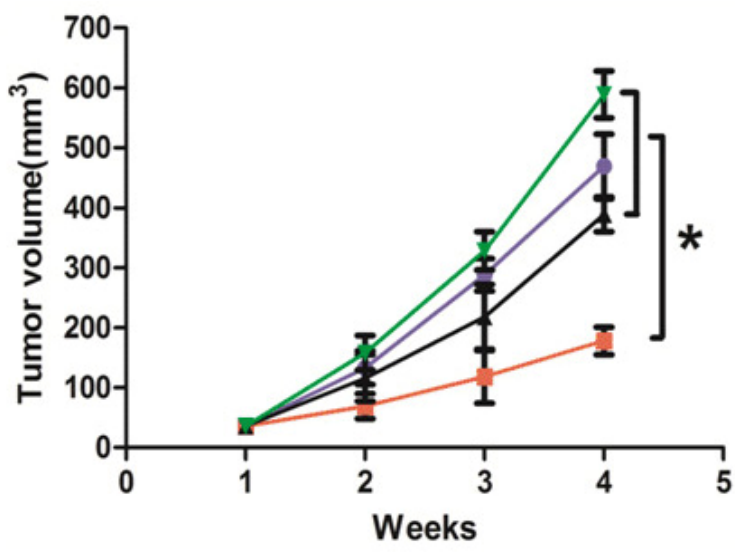

sh-MTA1+DMSO sh-MTA1+CDDP sh-Ctrl+CDDP sh-Ctrl+DMSO
Fig. 4. MTA1 knockdown inhibites NPC tumorigenesis and increases CDDP sensitivity in CNE1/CDDP xenograft tumor-harbored nude mice. (A) The tumor growth rate was inhibited when MTA1 was knocked down, especially combined the use of CDDP. (B) The size of the xenografts derived from CNE1/CDDP sh-MTA1 cells were much smaller than that from sh-Ctrl treated cells after receiving the same doses of CDDP or CNE1/CDDP shMTA1 cells treated with DMSO.

B

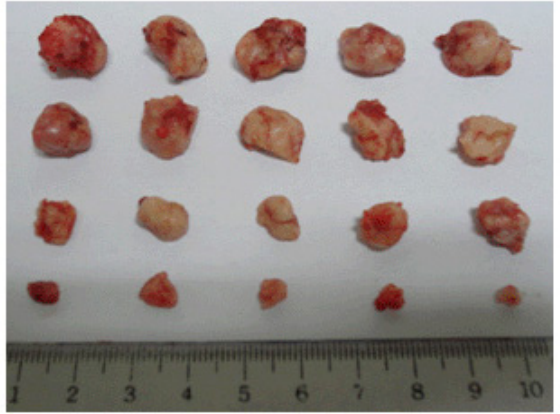

sh-Ctrl+DMSO

sh-MTA1+DMSO

sh-Ctrl+CDDP

sh-MTA1+CDDP

acquired cisplatin resistance (Godwin et al., 2013). Previously, it is demonstrated that MTA1 is a directtarget of the NF-kB signaling pathway (Bui-Nguyen et al., 2010). We speculated that the NF- $\mathrm{KB}$ signaling pathway may mediate the induction of MTA1 in CDDP-resistant NPC cells.

It is interesting that whether MTA1 is specific for resistance to CDDP or other chemotherapeutic drugs, such as doxorubicin (DOX)or cyclophosphamide (CTX). In the present study, we found that there was no significant difference between CNE1 and MTA1-overexpressing CNE1 cells in DOX or CTX resistance (data not shown). These results suggest that MTA1 may be specific for resistance to CDDP in NPC cells. It will be important to determine whether our findings are widespread in various cancer cells.

In summary, our study indicate that MTA1 confers CDDP resistance in NPC cells probably via PI3K/Akt pathway-dependent CSCs. MTA1 may be a potential target for overcoming CDDP resistance in NPC therapy.

\section{ACKNOWLEDGMENTS}

This work was supported by Research Project of Guangzhou Medical University (2013C61).

\section{REFERENCES}

Barr, M.P., Gray, S.G., Hoffmann, A.C., Hilger, R.A., Thomale, J., O'Flaherty, J.D., Fennell, D.A., Richard, D., O'Leary, J.J., and O'Byrne, K.J. (2013). Generation and characterisation of cisplatin-resistant non-small cell lung cancer cell lines displaying a stem-like signature. PLoS One 8, e54193.

Bui-Nguyen, T.M., Pakala, S.B., Sirigiri, R.D., Xia, W., Hung, M.C. Sarin, S.K., Kumar, V., Slagle, B.L., and Kumar, R. (2010). NFkappaB signaling mediates the induction of MTA1 by hepatitis B virus transactivator protein HBx. Oncogene 29, 1179-1189.
Donnenberg, V.S., and Donnenberg, A.D. (2005). Multiple drug resistance in cancer revisited: the cancer stem cell hypothesis. J.Clin.Pharmacol. 45, 872-877.

Godwin, P., Baird, A.M., Heavey, S., Barr, M.P., O'Byrne, K.J., and Gately, K. (2013). Targeting nuclear factor-kappa B to overcome resistance to chemotherapy. Front.Oncol. 3, 120.

Kumar, R., Wang, R.A., and Bagheri-Yarmand, R. (2003). Emerging roles of MTA family members in human cancers. Semi.Oncol. 30, 30-37.

Kumar, R., Balasenthil, S., Pakala, S.B., Rayala, S.K., Sahin, A.A., and Ohshiro, K. (2010). Metastasis-associated protein 1 short form stimulates Wnt1 pathway in mammary epithelial and cancer cells. Cancer Res. 70, 6598-6608.

Li, D.Q., Pakala, S.B., Nair, S.S., Eswaran, J., and Kumar, R. (2012a). Metastasis-associated protein 1 /nucleosome remodeling and histone deacetylase complex in cancer. Cancer Res. 72, 387-394.

Li, Y., Huang, W., Huang, S., Du, J., and Huang, C. (2012b). Screening of anti-cancer agent using zebrafish: comparison with the MTT assay. Biochem.Biophys.Res.Commun. 422, 85-90

Liu, A.Y., Cai, Y., Mao, Y., Lin, Y., Zheng, H., Wu, T., Huang, Y., Fang X., Lin, S., Feng, Q., et al. (2014). Twist2 promotes self-renewal of liver cancer stem-like cells by regulating CD24. Carcinogenesis 35, 537-545.

Ma, B.B., Lui, V.W., Hui, E.P., Lau, C.P., Ho, K., Ng, M.H., Cheng, S.H., Tsao, S.W., and Chan, A.T. (2010). The activity of mTOR inhibitor RAD001 (everolimus) in nasopharyngeal carcinoma and cisplatin-resistant cell lines. Invest. New Drugs 28, 413-420.

Ma, L., Zhang, G., Miao, X.B., Deng, X.B., Wu, Y., Liu, Y., Jin, Z.R., Li, X.Q., Liu, Q.Z., Sun, D.X., et al. (2013). Cancer stem-like cell properties are regulated by EGFR/AKT/beta-catenin signaling and preferentially inhibited by gefitinib in nasopharyngeal carcinoma. FEBS J. 280, 2027-2041.

Morais, C., Gobe, G., Johnson, D.W., and Healy, H. (2010). Inhibition of nuclear factor kappa $B$ transcription activity drives a synergistic effect of pyrrolidine dithiocarbamate and cisplatin for treatment of renal cell carcinoma. Apoptosis 15, 412-425.

Nagaraj, S.R., Shilpa, P., Rachaiah, K., and Salimath, B.P. (2013). Crosstalk between VEGF and MTA1 signaling pathways contrib- 
ute to aggressiveness of breast carcinoma. Mol. Carcinog. [Epub ahead of print].

Reddy, S.D., Pakala, S.B., Molli, P.R., Sahni, N., Karanam, N.K Mudvari, P., and Kumar, R. (2012). Metastasis-associated protein 1/histone deacetylase 4-nucleosome remodeling and deacetylase complex regulates phosphatase and tensin homolog gene expression and function. J. Biol.Chem. 287, 27843-27850.

Salot, S., and Gude, R. (2013). MTA1-mediated transcriptional repression of SMAD7 in breast cancer cell lines. Eur. J.Cancer 49, 492-499.

Serin, M., Erkal, H.S., and Cakmak, A. (1999). Radiation therapy and concurrent cisplatin in management of locoregionally advanced nasopharyngeal carcinomas. Acta Oncol. 38, 1031-1035.

Shafee, N., Smith, C.R., Wei, S., Kim, Y., Mills, G.B., Hortobagyi, G.N., Stanbridge, E.J., and Lee, E.Y. (2008). Cancer stem cells contribute to cisplatin resistance in Brca1/p53-mediated mouse mammary tumors. Cancer Res. 68, 3243-3250.

Singh, S.K., Clarke, I.D., Terasaki, M., Bonn, V.E., Hawkins, C. Squire, J., and Dirks, P.B. (2003). Identification of a cancer stem cell in human brain tumors. Cancer Res. 63, 5821-5828.

Song, Q., Zhang, H., Wang, M., Song, W., Ying, M., Fang, Y., Li, Y., Chao, Y., and Zhu, X. (2013). MTA1 promotes nasopharyngea carcinoma growth in vitro and in vivo. J.Exp.Clin.Cancer Res. 32, 54.

Spano, J.P., Busson, P., Atlan, D., Bourhis, J., Pignon, J.P., Esteban, C., and Armand, J.P. (2003). Nasopharyngeal carcinomas: an update. Eur. J.Pharmacol. 39, 2121-2135.

Venkatesha, V.A., Parsels, L.A., Parsels, J.D., Zhao, L., Zabludoff, S.D., Simeone, D.M., Maybaum, J., Lawrence, T.S., and Morgan, M.A. (2012). Sensitization of pancreatic cancer stem cells to gemcitabine by Chk1 inhibition. Neoplasia 14, 519-525.

Vinogradov, S., and Wei, X. (2012). Cancer stem cells and drug resistance: the potential of nanomedicine. Nanomedicine 7, 597615.

Wang, H., Zhang, G., Zhang, H., Zhang, F., Zhou, B., Ning, F., Wang, H.S., Cai, S.H., and Du, J. (2014). Acquisition of epithelialmesenchymal transition phenotype and cancer stem cell-like properties in cisplatin-resistant lung cancer cells through AKT/beta-catenin/Snail signaling pathway. Eur. J. Pharmacol. 723, 156-166.

Zhang, Y., Ng, H.H., Erdjument-Bromage, H., Tempst, P., Bird, A. and Reinberg, D. (1999). Analysis of the NuRD subunits reveals a histone deacetylase core complex and a connection with DNA methylation. Genes Dev. 13, 1924-1935. 